\title{
JUURNAL.RU
}

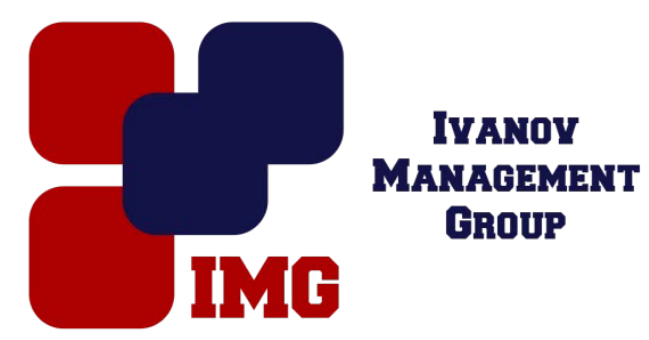

Бойко Т.А., Гордеева Н.М. Московский государственный технический университет им. Н.Э. Баумана Москва, Россия

doi: 10.18411/lj-30-04-2017-1-05

idsp 000001:1j-30-04-2017-1-05

\section{Оптимизация производственных процессов с использованием транспортной задачи}

\section{Аннотация}

В настоящей статье рассмотрено использование решения транспортной задачи для оптимального распределения работы между сотрудниками предприятия. В качестве критерия оптимальности выбрана максимизация производительности труда. Проанализирована последовательность решения транспортной задачи. Использовано три метода поиска первоначального опорного плана: метод северо-западного угла, метод минимального элемента, метод Вогеля. Для проверки опорного плана на оптимальность применен метод потенциалов. Показано, что проведенная оптимизация позволяет повысить производительность труда на 5 \%.

Ключевые слова: линейное программирование, оптимизационная задача, транспортная задача, задача о назначениях, опорный план, метод потенциалов.

\section{Abstract}

This article considers transportation problem solving for an optimal work allocation among corporate employees. Maximization of labour efficiency is used as an optimality criterion. Solution sequence of a transportation problem is analyzed. Initial basis is searched using three methods: northwest corner method, least element method and Vogel's method. Method of potentials is used to check initial basis for optimality. It is shown that considered optimization provides $5 \%$ increase of labor efficiency.

Keywords: linear programming, optimization problem, transportation problem, assignment problem, basis, method of potentials. 


\section{Введение}

В XXI веке в условиях ограниченности ресурсов - природных, временных, человеческих - возрастает потребность грамотного их использования. В связи с этим наиболее актуальными задачами математического моделирования экономических процессов являются оптимизационные задачи, то есть задачи, решение которых есть отыскание переменных, удовлетворяющих определённым условиям и обращающих в максимум (минимум) целевую функцию.

При этом оптимальным решением является то, которое удовлетворяет условиям заказчика исследования экономического процесса. Поэтому основной целью математика-экономиста, взявшегося за это исследование, будет расчетноколичественное обоснование итоговых результатов. При этом заказчик отставляет за собой право при принятии окончательного решения учитывать или не учитывать математические данные, полученные входе исследования.

Следует отметить, что в МГТУ им. Н.Э. Баумана уделяется большое внимание подготовке специалистов в области управления. Студентов знакомят с основами линейного программирования, которые в будущем помогут им решать задачи оптимизации при управлении производством на практике [3, 4].

\section{Постановка задачи}

Пусть имеется некоторая фирма $\mathrm{N}$, которая занимается разработкой программного обеспечения. Руководитель этой фирмы нанимает консультанта с целью изучения, анализа и поиска возможных путей увеличения производительности труда в компании.

При этом имеются следующие исходные данные.Всех сотрудников компании можно разделить на три группы:

1. Исполнители - сотрудники со специальным образованием; выполняют рутинную работу и тестирование продукта.

2. Специалисты - сотрудники с опытом и высшим образованием; способны выполнять любую работу.

3. «Звезды» - гениальные специалисты, которые готовы выполнять любую работу.

Производственный процесс состоит из 5 этапов:

1. Заключение договора с заказчиком.

2. Создание структуры, планирование производства.

3. Рутинные действия.

4. Тестирование составленных модулей.

5. Составление документации, подписание акта. 
В таблице 1 приведена информация о том, какие сотрудники могут выполнять конкретные этапы работ: «0» - сотрудник не способен выполнять данный тип работы, «1»- способен.

Стоит отметить, что первый этап работы - переговоры с заказчиком и заключение договора - проводят самые компетентные сотрудники компании «Звезды». На втором этапе к ним присоединяются Специалисты, которые составляют план проекта. Основную и самую затратную по времени часть работы выполняют Исполнители. Также они занимаются отладкой производственных модулей. Заключительную часть работы - составление отчетной документации - выполняют Специалисты так как этот этап требует определенной квалификации и опыта.

\begin{tabular}{|c|c|c|c|}
\hline \multicolumn{3}{|c|}{ Таблиц Сотрудники } \\
\cline { 2 - 4 } Этапы работ & Исполнители & Специалисты & «Звезды» \\
\hline Заключение договора & 0 & 1 & 1 \\
\hline Составление плана & 0 & 1 & 1 \\
\hline Рутинные действия & 1 & 1 & 1 \\
\hline Тестирование & 1 & 1 & 1 \\
\hline Завершающие действия & 0 & 1 & 1 \\
\hline
\end{tabular}

\section{Формирование качественных и количественных показателей}

Математическая модель всегда жертвует точностью. Во-первых, в данной задаче рассматриваются однотипные, равномерно распределенные по времени заказы. Во-вторых, руководство пока не планирует сокращать, нанимать или обучать персонал. Поэтому мы можем считать, что в компании работает постоянное количество сотрудников с конкретной квалификацией. Следовательно, и фонд оплаты труда не изменяется.

Консультанту необходимо оценить производительность труда в компании. Для этого ему необходимо иметь представление об основных понятиях Научной школы управления в менеджменте, в частности -о хронометраже трудовых операций. Консультант должен рассчитать, сколько времени тратит каждый сотрудник на выполнение конкретной работы. Также необходимо выполнить анализ внутренней среды компании. Консультанту следует выделить основные срезы фирмы: кадровый, организационный, производственный, маркетинговый и финансовый - проанализировать и определить их слабые и сильные стороны. Сбор таких данных является очень ответственным этапом работы, требующим аккуратности, скрупулёзности и опыта выполняющего. Стоит понимать, что при неправильном анализе потенциала фирмы поставленная задача может быть 
решена правильно с математической точки зрения, но при этом это решение не найдет применения на практике.

Учитывая вышесказанное, можно воспользоваться моделью транспортной задачи, которая решается с помощью теории линейного программирования [5]. Для правильного понимания последующего изложения решения задачи стоит отметить, что вместо стандартных полей транспортной задачи «Склады» и «Потребители», мы будем рассматривать «Этапы работ» и «Группы сотрудников». Вместо стоимости перевозки мы учитываем коэффициенты производительности труда, полученные на этапе сбора информации. Стоит обратить внимание на то, что целевая функция стандартной транспортной задачи должна быть минимизирована. Но нашей целью является максимизирование производительности труда. Поэтому необходимо поменять знаки при коэффициентах в целевой функции - в транспортной таблице 2 все они записаны со знаком «-».

Следует отметить, что в теории оптимизации выделена задача о назначениях, являющаяся частным случаем транспортной задачи [1]. Ее решение направлено на распределение $\mathrm{n}$ сотрудников на пвакантных мест так, чтобы общая прибыль фирмы после назначения была наибольшей. В нашей ситуации можно было воспользоваться решением этой задачи. Но целесообразно произвести все математические операции с помощью решения транспортной задачи, так как коллектив компании содержит сотрудников, способных выполнять несколько этапов работы, а потому рационально распределить стадии проекта между разными группами сотрудников.

\section{Решение задачи. Постановка транспортной задачи}

Имеется $m=5$ этапов работ, которые необходимо выполнить, используя в трудовом процессе $n=3$ групп сотрудников.Обозначения:

- $a_{i}>0$ - количество единиц работы на і-ом этапе;

- $b_{j}>0$ - количество сотрудников ј-ой группы;

- $c_{i j}>0$ - производительность работы на і-ом этапе сотрудником j-ой группы.

Требуется спланировать распределение обязанностей, которое удовлетворяет следующим условиям:

- выполнены все этапы работы в полном объеме;

- все сотрудники предприятия вовлечены в трудовой процесс; 
- производительность выполняемых работ должна быть максимальной.

План производительности есть матрица вида:

$$
X=\left(\begin{array}{cccc}
x_{11} & x_{12} & \ldots & x_{1 n} \\
x_{21} & x_{22} & \ldots & x_{2 n} \\
\ldots & \ldots & \ldots & \ldots \\
x_{m 1} & x_{m 2} & \ldots & x_{m n}
\end{array}\right)
$$

где $x_{i j}$ - объем работ i-го этапа, выполняемый сотрудником ј-ой группы.

Математическая модель сформулированной задачи имеет вид:

$$
\begin{aligned}
& f(x)=\sum_{i=1}^{m} \sum_{j=1}^{n} c_{i j} \cdot x_{i j} \rightarrow \max \\
& \sum_{j=1}^{n} x_{i j}=a_{i}, \quad i=\overline{1, m} ; \\
& \sum_{i=1}^{m} x_{i j}=b_{j}, \quad j=\overline{1, n} ; \\
& x_{i j} \geq 0 .
\end{aligned}
$$

Целевая функция (1) описывает суммарную производительность всех сотрудников на каждом этапе работы. Равенства (2) и (3) отражают требования того, что вся работа должна быть выполнена, и весь коллектив должен быть задействован при ее выполнении. Неравенство (4) является тривиальным, учитывая то, что объем работ должен быть неотрицательным.

Наша задача является закрытой, так как выполнено условие баланса:

$$
\sum_{i=1}^{n} a_{i}=\sum_{j=1}^{m} b_{j}=100
$$

Матрица производительности размерности $m \times n$ является допустимой, если удовлетворяет условиям (2) - (4). Для нашей задачи допустимая матрица будет оптимальной, если при ее значениях будет достигаться максимум целевой функции.

Число переменных $x_{i j}$ равнот $\cdot n$, а число уравнений в условиях (2) - (3) $m+n$. При этом всегда одно уравнение можно выразить из условия (5). Таким образом, мы получаем $m+n-1$ линейно независимых уравнений. Следовательно, общее число базисных переменных должно равняться $m+n-$ 1. 
Составим математическую модель транспортной задачи, исходные данные которой представлены в таблице 2.

\begin{tabular}{|c|c|c|c|c|}
\hline Этапы работы & Исполнители & Специалисты & «Звезды» & \multirow{2}{*}{$\begin{array}{c}\text { Всего } \\
\text { единиц } \\
\text { работы } \\
5\end{array}$} \\
\hline Заключение договора & 0 & -8 & -10 & \\
\hline Составление плана & 0 & -5 & -5 & 10 \\
\hline Рутинные действия & -10 & -6 & -2 & 50 \\
\hline Тестирование & -1 & -6 & -3 & 20 \\
\hline Завершающие работы & 0 & -5 & -10 & 15 \\
\hline Всего сотрудников & 60 & 30 & 10 & 100 \\
\hline
\end{tabular}

Целевая функция задачи определяется произведением $x_{i j}$-го объема работ на $c_{i j}$-ую производительность, то есть:

$$
\begin{aligned}
f(x)= & 0 \cdot x_{11}+(-8) \cdot x_{12}+(-10) \cdot x_{13}+0 \cdot x_{21}+(-5) \cdot x_{22}+(-5) \cdot x_{23} \\
& +(-10) \cdot x_{31}+(-6) \cdot x_{32}+(-2) \cdot x_{33}+(-1) \cdot x_{41}+(-6) \cdot x_{42} \\
& +(-3) \cdot x_{43}+0 \cdot x_{51}+(-5) \cdot x_{52}+(-10) \cdot x_{53} .
\end{aligned}
$$

Эта функция по условию задачи должна быть максимизирована.Запишем условия ограничений:

- по строкам таблицы:

- по столбцам таблицы:

$$
\begin{aligned}
& x_{11}+x_{12}+x_{13}=5, \\
& x_{21}+x_{22}+x_{23}=10 \\
& x_{31}+x_{32}+x_{33}=50 \\
& x_{41}+x_{42}+x_{43}=20 \\
& x_{51}+x_{52}+x_{53}=15 .
\end{aligned}
$$

$$
\begin{aligned}
& x_{11}+x_{21}+x_{31}+x_{41}+x_{51}=60 \\
& x_{12}+x_{22}+x_{32}+x_{42}+x_{52}=30 \\
& x_{13}+x_{23}+x_{33}+x_{43}+x_{53}=10
\end{aligned}
$$

Необходимо также учесть неравенство (4):

$$
x_{i j} \geq 0, i=\overline{1, m}, j=\overline{1, n} .
$$

Число базисных переменных равно: $m+n-1=5+3-1=7$.

При решении транспортных задач используют подход, состоящий из следующих этапов:

1. Нахождение опорного плана.

2. Проверка опорного плана на оптимальность.

2.1 План оптимален - задача решена.

2.2 План не оптимален - переходим к пункту 3.

3. Поиск оптимального плана.

Рассмотрим каждый из этих этапов в отдельности.

\section{Нахождение опорного плана}






Производительность труда равна:

$-f(x)=5 \cdot 0+10 \cdot 0+45 \cdot(-10)+5 \cdot(-6)+20 \cdot(-6)+5 \cdot(-5)+10 \cdot(-10)=-725$.

Метод минимального элемента

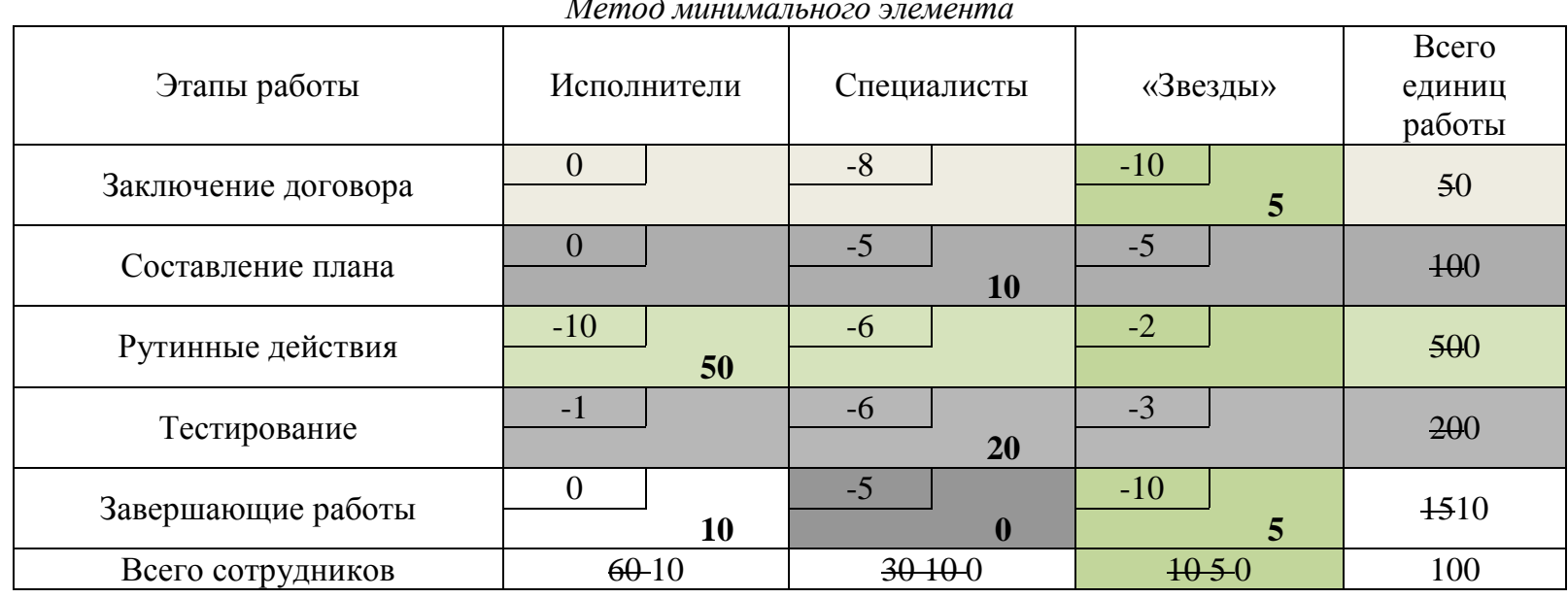

Производительность труда равна:

$$
\begin{aligned}
-f(x)=5 \cdot(-10)+10 \cdot(-5)+50 \cdot(-10)+20 \cdot(-6)+0 \cdot(-5)+5 \cdot(-10) \\
+10 \cdot 0=-770 .
\end{aligned}
$$

Таблицุа 5.

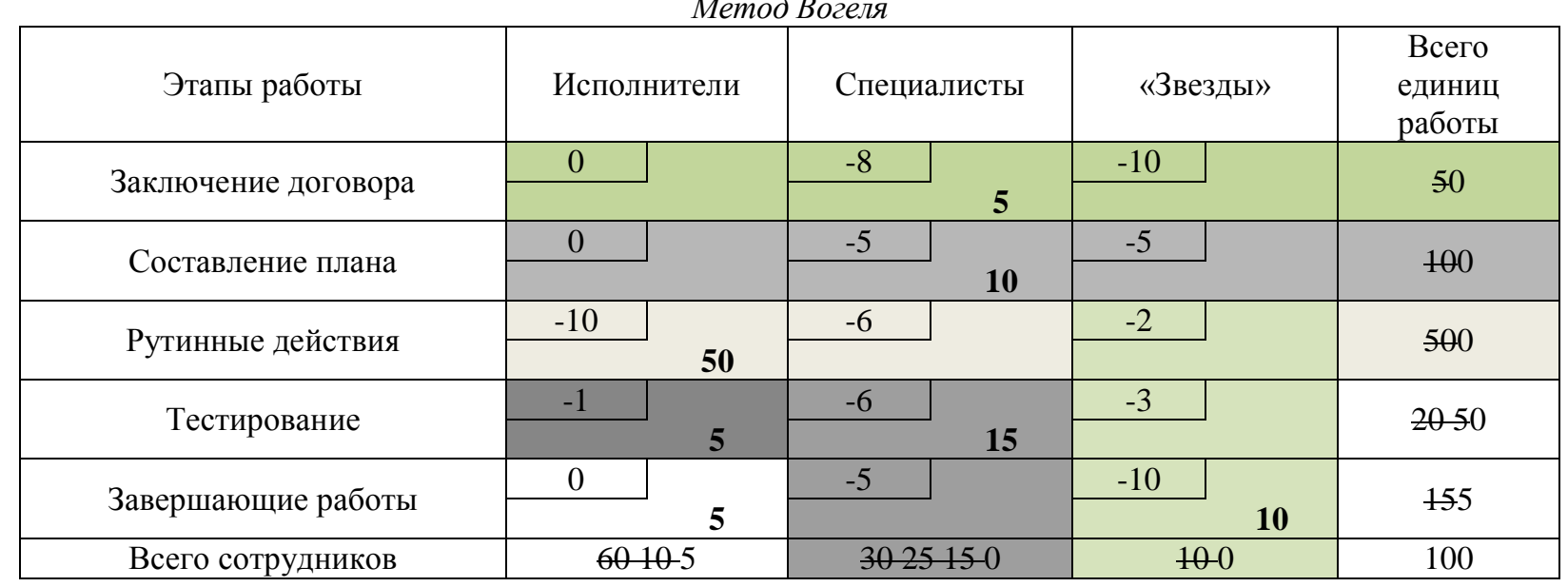

Производительность труда равна: 


$$
\begin{gathered}
-f(x)=5 \cdot(-8)+10 \cdot(-5)+50 \cdot(-10)+5 \cdot(-1)+15 \cdot(-6)+5 \cdot 0+10 \\
\cdot(-10)=-785 .
\end{gathered}
$$

Вывод: дальнейшее рассмотрение задачи будет вестись на основе опорного плана, полученного с помощью метода северо-западного угла.

\section{Проверка опорного плана на оптимальность}

Полученный первоначальный опорный план необходимо проверить на оптимальность, то есть убедиться в том, что данное решение задачи обеспечивает максимальную производительность.

Проверку осуществим с помощью метода потенциалов [2]. Под критерием оптимальности будем понимать тот случай, когда сумма соответствующих потенциалов каждой свободной ячейки таблицы будет больше либо равно производительности в этой ячейке.

Условия оптимальности:

- $U_{i}+V_{j}=C_{i j}$ - для базисных ячеек;

- $U_{i}+V_{j} \leq C_{i j}$ - для свободных ячеек.

Следует отметить, что при проверке опорного плана на оптимальность необходимо учесть дополнительные условия: Исполнители не могут выполнять 1,2 и 5 этапы работ (см. таблицу 1). Следовательно, нужно «запретить» назначать эту группу сотрудников на данные стадии проекта. Математически это можно представить следующим образом: руководителю компании должно быть невыгодно распределить работника на вышеуказанные этапы работ. Для этого производительность Исполнителей должна быть максимально низкой. Обозначим $C_{11}, C_{21}, C_{51}$ черезM, где $\mathrm{M}$ - бесконечно малое число.

Таким образом, получим таблицу 6.

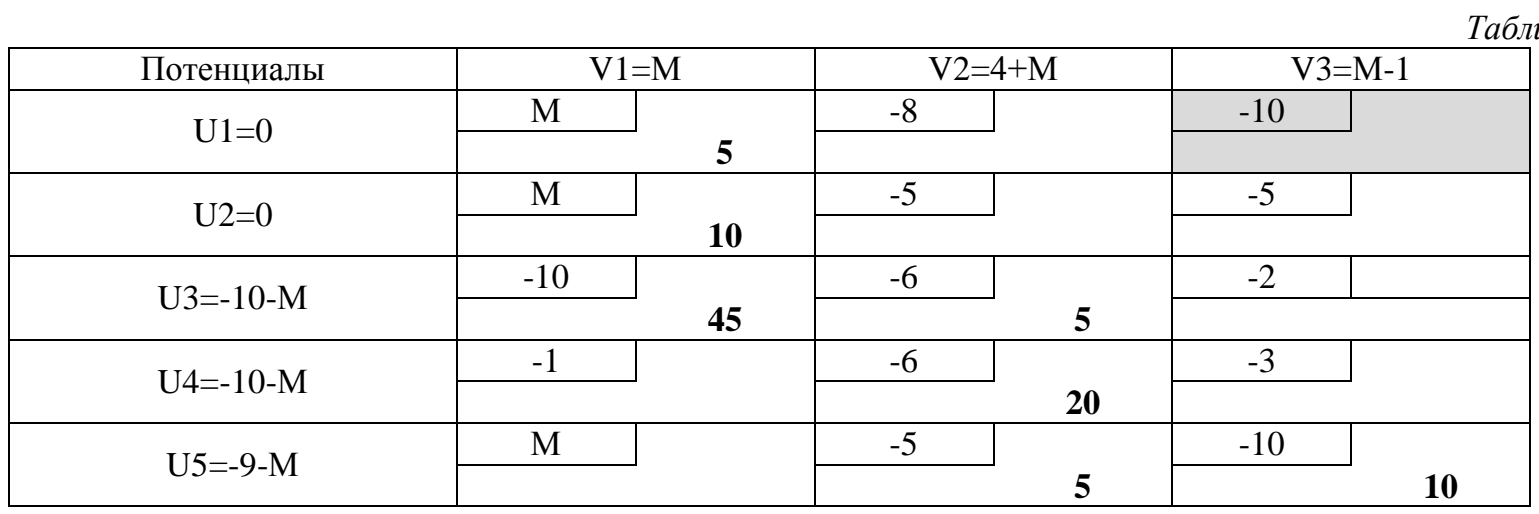

Рассчитаем потенциалы и проверим их для свободных клеток. В ячейке 13 условие оптимальности не выполнено, следовательно, необходимо найти оптимальное решение.

\section{Поиск оптимального плана}


Таблицуа 7.

Первая итерация



Таблица 8.

Вторая итераиия

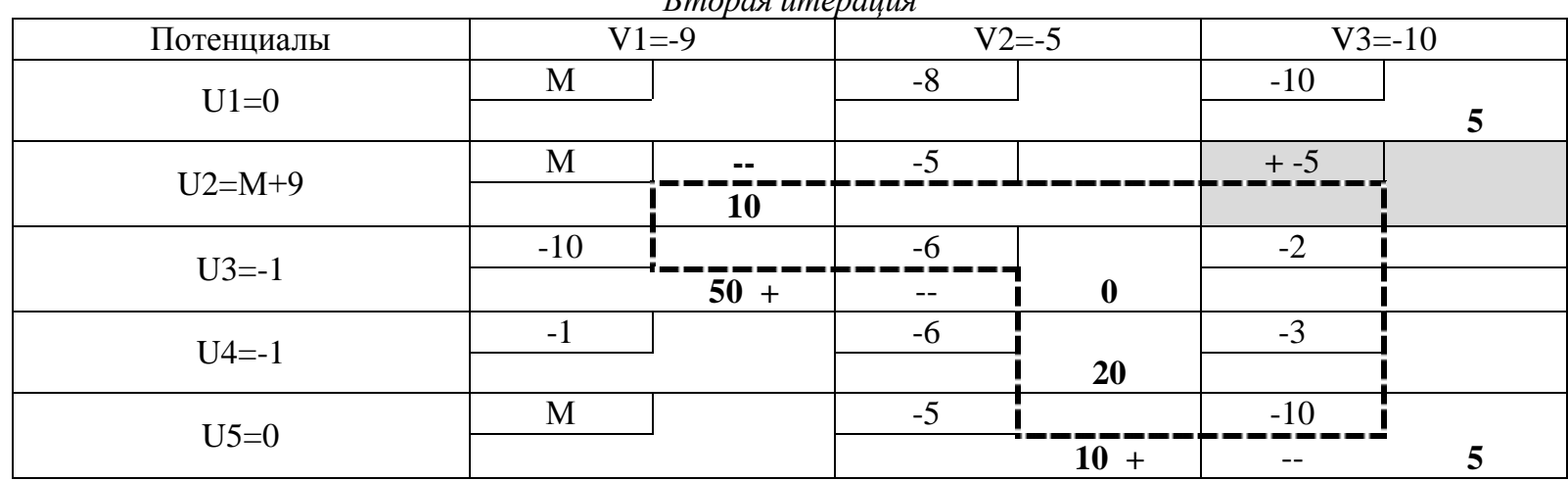

Таблица 9.

Третья итераичя

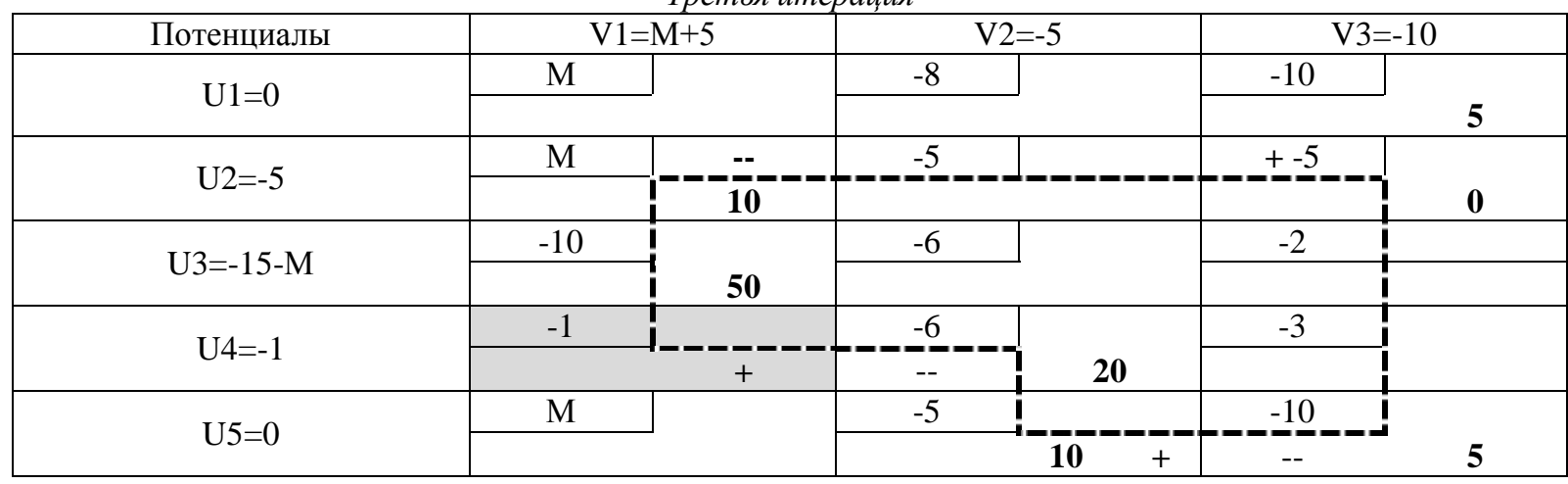

Четвертая итерация

\begin{tabular}{|c|c|c|c|c|c|c|}
\hline Потенциалы & \multicolumn{2}{|c|}{$\mathrm{V} 1=\mathrm{M}-5$} & \multicolumn{2}{|c|}{$\mathrm{V} 2=\mathrm{M}-10$} & \multicolumn{2}{|c|}{$V 3=-10$} \\
\hline \multirow{2}{*}{$\mathrm{U} 1=0$} & $\mathrm{M}$ & & -8 & & -10 & \multirow[b]{2}{*}{5} \\
\hline & & & & & & \\
\hline \multirow{2}{*}{$\mathrm{U} 2=5$} & $\mathrm{M}$ & -- & +-5 & & -5 & \multirow[b]{2}{*}{5} \\
\hline & & $\overline{5}$ & & & & \\
\hline \multirow{2}{*}{ U3=-5-M } & -10 & \multirow[b]{2}{*}{50} & \multirow[t]{2}{*}{-6} & & -2 & \\
\hline & & & & & & \\
\hline \multirow{2}{*}{$\mathrm{U} 4=4-\mathrm{M}$} & -1 & & -6 & \multirow[b]{2}{*}{15} & -3 & \\
\hline & & & -- & & & \\
\hline \multirow{2}{*}{$\mathrm{U} 5=5-\mathrm{M}$} & $\mathrm{M}$ & & -5 & \multirow[b]{2}{*}{15} & -10 & \\
\hline & & & & & & \\
\hline
\end{tabular}

Таблицฺа 11. 


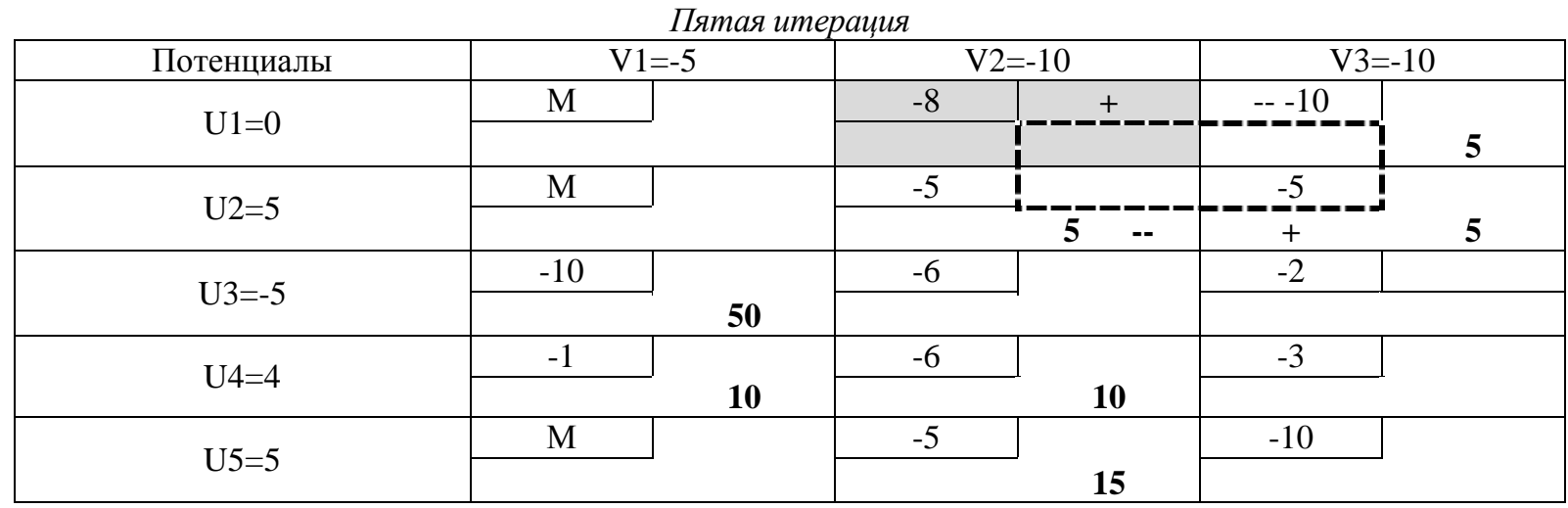

На пятой итерации мы достигли того, что все сотрудники компании выполняют только ту работу, которую могут выполнить в соответствии со своей квалификацией. Данные, представленные в таблице 11, получил консультант на этапе формирования качественных и количественных показателей. Теперь необходимо: с экономической точки зрения изменить расстановку кадров, а с математической - найти оптимальное решение, так как до сих пор не выполнено условие оптимальности в ячейке 12.

Производительность труда на пятой итерации равна:

$$
\begin{aligned}
-f(x)= & 5 \cdot(-10)+5 \cdot(-5)+5 \cdot(-5)+50 \cdot(-10)+10 \cdot(-1)+10 \cdot(-6) \\
& +15 \cdot(-5)=-745 .
\end{aligned}
$$

Таблица 12.

\begin{tabular}{|c|c|c|c|c|c|c|}
\hline Потенциалы & \multicolumn{2}{|c|}{$\mathrm{V} 1=-3$} & \multicolumn{2}{|c|}{$\mathrm{V} 2=-8$} & \multicolumn{2}{|c|}{$V 3=-8$} \\
\hline \multirow{2}{*}{$\mathrm{U} 1=0$} & $\mathrm{M}$ & & -8 & \multirow[b]{2}{*}{5} & -10 & \\
\hline & & & & & & \\
\hline \multirow{2}{*}{$\mathrm{U} 2=3$} & $\mathrm{M}$ & & -5 & + & ---5 & \multirow[b]{2}{*}{10} \\
\hline & & & & $\mathbf{0}$ & & \\
\hline \multirow{2}{*}{$\mathrm{U3}=-7$} & -10 & \multirow[b]{2}{*}{50} & -6 & & -2 & \\
\hline & & & & & & \\
\hline \multirow{2}{*}{$\mathrm{U} 4=2$} & -1 & \multirow[b]{2}{*}{10} & -6 & \multirow[b]{2}{*}{10} & -3 & \\
\hline & & & & & & \\
\hline \multirow{2}{*}{$\mathrm{U5}=3$} & $\mathrm{M}$ & & -5 & \multirow[b]{2}{*}{5} & -10 & \\
\hline & & & & & + & \\
\hline
\end{tabular}

Шестая итерацчия

Таблица 13.

Седьмая итерачия

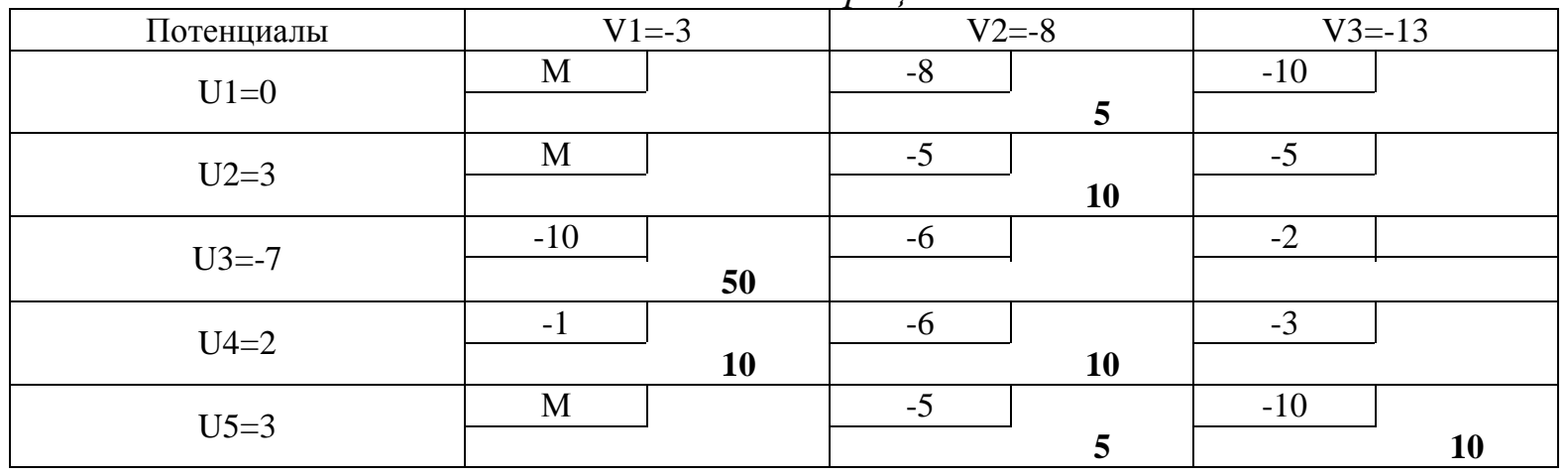


Найдено оптимальное решение.

Значение целевой функции равно:

$$
\begin{gathered}
-f^{*}(x)=5 \cdot(-8)+10 \cdot(-5)+50 \cdot(-10)+10 \cdot(-1)+10 \cdot(-6)+5 \cdot(-5) \\
+10 \cdot(-10)=-785 .
\end{gathered}
$$

Таким образом, производительность труда повысится на:

$$
k=\left|\frac{f^{*}(x)-f(x)}{f(x)}\right| \cdot 100 \%=\left|\frac{-785+745}{-745}\right| \cdot 100 \%=5 \% .
$$

\section{Анализ результатов и выводы}

Из итоговой таблицы (см. таблицу 13) видно, что требуются незначительные изменения. Руководитель должен поручить заключение договоров Специалистам, при этом отобрать эту работу у «Звезд». Этап составления плана также должен полностью перейти к Специалистам. Очень трудоемкая и требующая высокой квалификации работа по написанию сопровождающей документации должна в большей степени выполняться «Звездами». Ожидается, что при такой расстановке кадров производительность повысится на $5 \%$.

Следует еще раз отметить, что при рассмотрении данной задачи не учитывались определенные условии. Одним из них является время. В связи с этим можно предложить варианты возможного усложнения задачи.

Во-первых, стоит рассмотреть транспортную задачу по критерию времени. Бывают форс-мажорные ситуации, когда самым ограниченным ресурсом выступает время. Тогда вместо производительности следует рассматривать количество часов, необходимое для выполнения одной операции конкретным сотрудником. В этом случае кадровое распределения работников будет другим.

Во-вторых, можно проанализировать тот случай, когда будет уместна транспортная параметрическая задача. Возможно, она более точно сможет учесть риск такого важного этапа работы, как сбор показателей. В этом случае, что производительность, что время задаются не конкретным числом, а интервалом допустимых значений, в пределах которого колеблется интересующий нас параметр. С помощью этой задачи может быть разработан наиболее рациональный план размещения сотрудников при нестабильном временном запасе и до конца не изученных производственных мощностях.

Из всего вышесказанного можно сделать вывод о том, что в данной ситуации, при исключении из рассмотрения определенных условий, задача решена математически верно. В результате проведенной работы можно 
сэкономить один рабочий день в месяц, что является хорошим показателем эффективности решения.

1. Волков И.К., Загоруйко Е.А. Исследование операций: Учеб. для вузов. - 3 е изд., стереотип. / Под ред. В.С. Зарубина, А.П. Крищенко. - М.: Изд-во МГТУ им. Н.Э. Баумана, 2004. - 440 с. (Сер. Математика в техническом университете; Вып. XX). ISBN 5-7038-1518-5 (Вып. XX); ISBN 5-7038-1270-4.

2. Галеев Э.М. Оптимизация: Теория, примеры, задачи: Учебное посо-бие. Изд. 6-е, испр. - М.: ЛЕНАНД, 2015. - 344 с.

3. Гордеева Н.М., Самойлова И.А. Использование прикладных задач в обучении математике будущих менеджеров // Современные проблемы науки и образования. 2016. - № 2. - С. 233.

4. Гордеева Н.М., Самойлова И.А. Применение исследования операций к моделированию инженерных задач // В книге: Управление качеством инженерного образования. Возможности ВУЗов и потребности промышленности. Тезисы докладов второй международной научно-практической конференции. - 2016. - С. 41-42.

5. Исследование операций в экономике: учебник для академического бакалавриата / Н.Ш. Кремер, Б.А. Путко, И.М. Тришин, М.Н. Фридман; под ред. Н.Ш. Кремера. - 3-е изд., перераб. и доп. - М.: Издательство Юрайт, 2016. - 438 с. - Серия: Бакалавр. Академический курс. ISBN 978-5-9916-3748-0. 